\title{
Up-regulation of BTLA expression in myeloid dendritic cells in neonatal sepsis associated with the treatment outcome and down-regulation of DC function
}

\section{Wandang Wang}

Xiaolan Hospital Affiliated to Southern Medical University

Xuran Yang

Southern Medical University Nanfang Hospital

Mingfa Guo

Children's Hospital Affiliated to Zhengzhou University

Zhifeng Pan

Xiaolan Hospital Affiliated to Southern Medical University

Mingjin Qiu

Xiaolan Hospital Affiliated to Southern Medical University Jingyi Wu

Xiaolan Hospital Affiliated to Southern Medical University

Jing Jia

Children's Hospital Affiliated to Zhengzhou University

Yingliang Liang

Xiaolan Hospital Affiliated to Southern Medical University Wenting Zheng

Xiaolan Hospital Affiliated to Southern Medical University Guanghui Chen ( $\nabla$ chenguanghui416@sina.com )

Southern Medical University Affiliated Xiaolan Hospital https://orcid.org/0000-0002-7212-9490

Research

Keywords: BTLA; myeloid dendritic cells; neonatal sepsis

Posted Date: March 17th, 2020

DOI: https://doi.org/10.21203/rs.3.rs-17398/v1

License: @ (i) This work is licensed under a Creative Commons Attribution 4.0 International License. Read Full License 


\section{Abstract}

Background: Neonatal sepsis is an acute life-threatening condition in neonates, and a proper innate inflammatory is essential for prevention of the systemic inflammation associated with sepsis. As the most potential antigen-presenting innate immune cells, dentritic cells (DCs) dysfunction has been verified detrimental for sepsis. B and T lymphocyte attenuator (BTLA) is an immune-regulatory receptor shown to be associated with DCs dysfunction. However, the role of BTLA expression in myeloid DCs (mDCs) in neonatal sepsis is unknown.

Methods: 61 of neonates with sepsis and 32 of neonates having no suspicion of sepsis as control were enrolled into this study. BTLA and HLA-DR expression in mDCs was measured by flow cytometry. To further study the role of BTLA in regulating mDCs function, BTLA+mDCs and BTLA-mDCs from septic neonates were sorted and utilized to evaluate the phagacytosis capacity, bactericidal ability as well as cytokine secretion of mDCs.

Results: A higher percentage of BTLA+mDCs were observed in neonatal septic patients and the percentage was positively correlated to the duration of hospitalization of neonates as well as the severity of sepsis. Moreover, a decrease MFI expression of HLA-DR was found in mDCs in neonatal sepsis, which expression was negatively correlated with the percentage of BTLA+mDCs. When compared to BTLA-mDCs, sorted BTLA+mDCs exhibited lower FITC-dextran uptake capacity but more CFU E.coli number after cells challenged by E.coli. In addition, BTLA+mDCs comparatively secreted lower level of TNF-a and IL-12, but higher IL-10.

Conclusions: A higher level of BTLA in mDCs in the observed septic neonates was associated to the severity of neonatal sepsis; therefore, BTLA expression in mDCs could be a useful biomarker help to determine the neonatal sepsis development. Additionally, BTLA negatively regulated the phagocytosis capacity and bactericidal ability of $\mathrm{mDCs}$ and lowered their antigen-presenting ability as well as altered cells into an anti-inflammatory phenotype. Thus, targeting BTLA in mDCs may be a new therapeutic strategy for neonatal sepsis.

\section{Background}

Neonatal sepsis is an acute life-threatening condition in neonates caused by the infection of pathogens. Despite the advances of neonatal care, sepsis still remains the third leading causes of neonatal death in worldwide, especially in developing countries [1, 2]. An updated analysis indicated that, of 6.3 million deaths under the age of 5 years in 2013, 51.8\% (3.257 million) were attributable to infectious causes and $44 \%$ ( 2.761 million) died in neonatal period [3]. Host defense pathogen infection is dependent on coordinated innate and adaptive immune system. However, Immune dysfunction is often accompanied with the progression of sepsis, and the prognosis of sepsis is largely dependent on the immune status of the host, as the initial over-stimulation of immune cells responses and subsequent immunosuppression leads to the multi-organ failure and even death in neonates [4-6]. Therefore, by better understanding the response profiles of various immune cells during sepsis, we will be able to target their responses to reduce or prevent the occurrence of neonatal sepsis. Dendritic cells (DCs), the most potential antigen-presenting cells, serve as barometers of the immune response against infection by determining the inflammatory milieu and promoting the initiation of adaptive immune responses. It has been found dysfunction of myeloid DCs (mDCs) was detrimental for sepsis [7-9]. The dysfunction of DCs could lead to an immunosuppressive environment by inducing high level of IL-10 and TGF- $\beta$ which causes an upregulation of immunosuppressive regulatory T cells (Tregs) along with an impaired adaptive immune cell responses[10]. However, whether the dysfunction of $\mathrm{mDC}$ involved in the development of neonatal sepsis has still yet to be elucidated.

$B$ and T lymphocyte attenuator (BTLA), an inhibitory co-receptor belonging to the CD28 family, its expression on DCs has been found associates with the DCs dysfunction [11, 12]. BTLA was firstly found expressed in CD4 ${ }^{+} T$ cells and B cells with similarities to cytotoxic T-lymphocyte antigen-4 (CTLA-4) and programmed death-1 (PD-1)[13]. Thereafter, accumulating evidence verified that not only CD $4^{+} T$ cells and B cells, most lymphocyte and immune cells, including monocytes, macrophages, neutrophils, DCs, NK cells as well as CD8 ${ }^{+} T$ cells, also appear to be induced to express BTLA and the ligation of BTLA may have effects on them as well [14]. BTLA acts as a ligand of herpesvirus entry mediator (HVEM; TNFRSF14), a TNFR super family member found on T, B, NK, DC and myeloid cells [15]. The interaction of BTLA and HVEM can govern T cell responses, including their memory and regulation functions [15-18]. Prior study demonstrated that $\mathrm{BTLA}^{-/-}$mice exhibit significantly higher bacterial clearance compared with WT mice in the early phase of bacterial infection[19]. Shubin et al. [20] have also shown that BTLA ${ }^{-/-}$mice exhibited a higher rate of survival and protection from cecal ligation and puncture (CLP). Moreover, recent studies showed that high expression of BTLA on the surface of DCs could negatively regulate their anti-tuberculosis immune activity in pleural tuberculosis patients $[21,22]$. These findings suggest that BTLA is involved in the clearance of pathogens in the early phase of immune responses and BTLA expression on innate cells might be involved in the process. However, whether the BTLA expression on mDCs is associated with the sepsis in neonates patients remains to be elucidated.

In this study, we collected clinical samples of neonates with sepsis to determine the regulation role of BTLA on mDCs and the association of neonatal sepsis with BTLA-expressing mDCs, importantly, to determine how BTLA expression mediated mDCs dysfunction that contributes to neonatal sepsis occurrence. Our data showed that the expression of BTLA in mDCs from neonatal patients was higher than that from non-septic neonates and a higher level of BTLA on mDCs positively correlated to a severe sepsis outcome in neonates. Further study demonstrated that BTLA expression could lower the phagocytosis ability and bactericidal capacity of $\mathrm{mDCs}$ and the potential mechanism maybe related to the regulation effect of BTLA on the expression of human leukocyte antigen-DR (HLA-DR) and the changes of cytokine secretion of mDCs.

\section{Materials And Methods}

\subsection{Patients}


The study was conducted between June 2018 and October 2019, which was approved by the ethical committee of the Xiaolan Hospital Affiliated to Southern Medical University and informed consent forms were signed by all guardian of participants. Neonatal sepsis inclusive criteria were: (1) Neonates (0-28 days) presented as body temperature abnormality, jaundice, and weak response; (2) Laboratory results showed blood leukocytes larger than $20 \times 10^{9}$ cells/L or smaller than $5 \times 10^{9}$ cells/L, rod nuclear cell ratio over 0.2 , and/or C-reactive protein (CRP) above $8.0 \mathrm{mg} / \mathrm{mL}$; and (3) Isolated pathogen from blood culture or the same opportunistic pathogen by 2 consecutive cultures. Neonates with significant clinical symptoms plus 2 of above abnormal laboratory results or positive blood cultures could be diagnosed as neonatal sepsis[23]. All patients received standard treatment for sepsis. Patient medical records including gestational ages, birth weight, delivery types, Apgar score at $1 \mathrm{~min}$, hematological parameters, pathogenic features, and the duration of hospitalization were reviewed. Each patient's data was recorded on a standardized data collection form. Populations in controls comprised neonates having no suspicion of sepsis but diagnosed with neonatal jaundice, hypoglycemia or wet lung.

\subsection{Blood routine examination and serological assays for infectious markers}

Whole blood samples from different groups were collected with EDTA- $\mathrm{K}_{2}$ anticoagulation tubes and procoagulant tube. The blood routine examination was processed with SysmexXN2000. Infectious markers, including procalcitonin (PCT), interleukin-6 (IL-6), CRP and serum amyloid A (SAA) were detected using assay kits in Roche Cobas-702 or Roche E411. All operations are performed in accordance with the SOP document and the manufacturer's protocols.

\subsection{Determination Of Btla Expression On Mdc}

The expression of BTLA on mDCs was determined by flow cytometry. Monoclonal antibodies Lin1-FITC, HLA-DR-PerCP, CD11c-PE and BTLA-APC were purchased from BD Biosciences (San Diego, CA, USA). 100 $\mu \mathrm{L}$ fresh collected whole blood of patients or controls was incubated with indicated antibodies for 30 minutes at $4{ }^{\circ} \mathrm{C}$ and then lysed with FACS ${ }^{\mathrm{TM}}$ lysing solution (BD Biosciences, San Jose, CA, USA).Subsequently, those stained samples were washed with phosphate buffered saline (PBS), fixed and eventually detected by BD FACS calibur with BD CellQuest software supporting. Data were analyzed using FlowJo software 7.6 (Tree Star Inc., San Carlos, CA, USA).

\subsection{Isolation And Purification Of Mdcs}

Peripheral blood monocytes cells (PBMCs) were isolated from freshly heparinized blood of neonatal sepsis using Ficoll-Histopaque (Sigma). Isolated PBMCs were incubated with indicated antibodies for 30 minutes at $4^{\circ} \mathrm{C}$ and lysed with FACS ${ }^{\mathrm{Tm}}$ lysing solution. Then, BTLA ${ }^{+} \mathrm{mDCs}^{\mathrm{and}} \mathrm{BTLA}^{-} \mathrm{mDC}$ populations were purified by flow sorting with BD FACS Ariall. The purity of the cells was $97.63 \%$.

\subsection{Fitc-dextran Uptake Assay}

The FITC-dextran uptake assay was setup by incubating cells with FITC-dextran in duplicate plates at $4{ }^{\circ} \mathrm{C}$ or $37^{\circ} \mathrm{C}$ as previously described [21]. Briefly, 5000 sorted $\mathrm{BTLA}^{+} \mathrm{mDC}$ s or BTLA${ }^{-} \mathrm{mDC}$ s were incubated with $1 \mathrm{mg} / \mathrm{mL}$ FITC-dextran in $5 \mathrm{~mL}$ Falcon ${ }^{\mathrm{Tm}}$ Polystyrene Round-Bottom Tubes (BD Biosciences) at $37^{\circ} \mathrm{C}$ and $4{ }^{\circ} \mathrm{C}$ (to determine baseline FITC-dextran uptake level) for $1 \mathrm{~h}$, respectively. Then, cells were washed twice with PBS and resuspended in $200 \mu \mathrm{L} 2 \%$ FBSPBS containing $2 \%$ paraformaldehyde before analyzed with BD FACS caliber flow cytometer. The percentage of phagocytosis was determined as following: Percentage of phagocytosis of $\mathrm{BTLA}^{+} \mathrm{mDCs}\left(\right.$ or BTLA $\left.{ }^{-} \mathrm{mDCs}\right)(\%)$ = Percentage of phagocytosis of $\mathrm{BTLA}^{+} \mathrm{mDCs}$ (or BTLA $\left.{ }^{-} \mathrm{mDCs}\right)$ at $37^{\circ} \mathrm{C}(\%)-\mathrm{Percentage}^{\circ}$ phagocytosis of $\mathrm{BTLA}^{+} \mathrm{mDCs}$ (or BTLA $\left.{ }^{-} \mathrm{mDCs}\right)$ at $4^{\circ} \mathrm{C}(\%)$.

\subsection{Bactericidal Activity Of Mdcs}

Sorted BTLA ${ }^{+} \mathrm{mDC}$ s or BTLA ${ }^{-} \mathrm{mDC}$ s from neonatal sepsis patients were cultured in 12-well plates at 5000 cells/well in RPMI 1640 medium (Gibco, Grand Island, NY, USA) and maintained at $37{ }^{\circ} \mathrm{C}$ in a humidified atmosphere of $5 \% \mathrm{CO}_{2}$. Afterwards, all groups of cells were infected with Escherichia coli (E.coli, ATCC15922) ( $\mathrm{mDCs} /$ bacteria ratio $=10: 1)$ in vitro for $6 \mathrm{~h}$. At indicated time, free bacteria were collected by Percoll gradient centrifugation (2000 rpm, $20 \mathrm{~min})$ and plated to $\mathrm{MH}$ agar to determine the bacteria killing capacity of $\mathrm{BTLA}^{+} \mathrm{mDCs}$ or $\mathrm{BTLA}^{-} \mathrm{mDCs}$.

\subsection{Cytokine Analysis}

By infected with E.coli for 6 h, cytokines in the supernatant of infection system were detected using ELISA kits. TNF-a, IL-12 and IL-10 were quantified using human TNF-a, IL-12 and IL-10 ELISA kit respectively (ABclonal). When cytokines in the culture supernatant were analyzed, the concentration (pg/mL) was determined and represented as mean \pm SEM.

\subsection{Statistical Analysis}

The data were expressed with mean \pm SEM. Flow cytometry data were analyzed by FlowJo software 7.6 (Tree Star Inc., San Carlos, CA, USA), and statistical analysis was carried out using GraphPad Prism 5 software (GraphPad Software Inc., La Jolla, CA, USA) by Student's t-test (parametric method) or MannWhitney test (non-parametric method). The correlation analysis was processed by Pearson correlation analysis. $\mathrm{P}$ values $<0.05$ was considered significant. 


\section{Results}

\subsection{Study Subject Characteristics}

A total 61 of neonates with clinical neonatal sepsis and 32 of neonates having no suspicion of sepsis were enrolled into this study. Significant difference were found between the two group patients including the Apgar score at $1 \mathrm{~min}$, duration of hospitalization, hematological parameters( including WBC, lymphocytes and neutrophil) and infectious markers( including PCT, IL-6, SAA and CRP). Other parameters such as gender, gestational ages, birth weight, mode of delivery and monocytes count had no statistical difference between two groups. Of 61 septic neonates, 40(65.57\%) has positive and $21(34.43 \%)$ has negative culture results. Among the 40 of culture positive cases, the pathogenic microorganism were 18(45\%) Gram-positive and 22(55\%) Gram-negative. Furthermore, data showed the infection were mainly distributed in blood (39,63.93\%), less distributed in other sites like lung $(12,19.67 \%)$, brain(7, 11.48\%), abdominal (2, 3.28\%) and $\operatorname{skin}(1,1.64 \%)($ Table 1$)$. 
Table 1

Clinical characteristics of the patients in this study

\begin{tabular}{|c|c|c|c|}
\hline Variable & Sepsis $(n=61)$ & Control $(n=32)$ & $\mathbf{P}$ \\
\hline Age(days) & $5.63 \pm 0.87$ & $4.33 \pm 0.93$ & $>0.05$ \\
\hline Gender(M/F) & $34 / 27$ & $15 / 17$ & $>0.05$ \\
\hline Gestational age (weeks) & $35.88 \pm 0.53$ & $37.09 \pm 0.31$ & $>0.05$ \\
\hline Preterm newborns/ Term newborns & $27 / 34$ & $12 / 20$ & $>0.05$ \\
\hline Birth weight (g) & $2620 \pm 120$ & $2890 \pm 120$ & $>0.05$ \\
\hline Delivery type (NVD/CS) & $32 / 29$ & $13 / 19$ & $>0.05$ \\
\hline Apgar score at $1 \mathrm{~min}$ & $9.05 \pm 0.22$ & $9.94 \pm 0.06$ & $<0.05$ \\
\hline EOS /LOS & $39 / 22$ & NA & \\
\hline Hospital length of stay (days) & $21.31 \pm 2.15$ & $7.28 \pm 0.82$ & $<0.05$ \\
\hline WBC count $\left(\times 10^{9}\right.$ cells/L) & $13.62 \pm 0.92$ & $10.93 \pm 0.48$ & $<0.05$ \\
\hline Lymphocyte count $\left(\times 10^{9}\right.$ cells/L) & $3.41 \pm 0.28$ & $4.29 \pm 0.23$ & $<0.05$ \\
\hline Monocytes count $\left(\times 10^{9}\right.$ cells/L) & $1.22 \pm 0.12$ & $1.01 \pm 0.05$ & $>0.05$ \\
\hline Neutrophil count $\left(\times 10^{9}\right.$ cells/L) & $8.72 \pm 0.76$ & $5.17 \pm 0.48$ & $<0.05$ \\
\hline Procalcitonin,PCT(ng/mL) & $16.59 \pm 2.89$ & $0.19 \pm 0.06$ & $<0.05$ \\
\hline Interleukin-6,IL-6(pg/mL) & $192.91 \pm 58.18$ & $9.09 \pm 2.37$ & $<0.05$ \\
\hline C-reactive protein,CRP(mg/L) & $21.66 \pm 3.73$ & $1.10 \pm 0.34$ & $<0.05$ \\
\hline Serum Amyloid A,SAA( mg/L ) & $19.1 \pm 21.36$ & $5.58 \pm 0.58$ & $<0.05$ \\
\hline \multicolumn{4}{|l|}{ Site of infection } \\
\hline Blood & $39(63.93 \%)$ & & \\
\hline Lung & $12(19.67 \%)$ & & \\
\hline Brain & $7(11.48)$ & & \\
\hline Abdominal & $2(3.28 \%)$ & & \\
\hline Skin & $1(1.64 \%)$ & & \\
\hline \multicolumn{4}{|l|}{ Microbiological diagnosis } \\
\hline Culture Negative & $21(34.43 \%)$ & & \\
\hline Culture Positive & $40(65.57 \%)$ & & \\
\hline Gram positive species & $18(45 \%)$ & & \\
\hline Group B streptococcus & $8(44.44 \%)$ & & \\
\hline Staphylococcus aureus & $2(11.11 \%)$ & & \\
\hline Staphylococcus haemolyticus & $2(11.11 \%)$ & & \\
\hline Staphylococcus epidermidis & $2(11.11 \%)$ & & \\
\hline Enterococcus Faecium & $2(11.11 \%)$ & & \\
\hline Pediococcus pentosaceus & $1(5.56 \%)$ & & \\
\hline Gram Negative species & $22(55 \%)$ & & \\
\hline Escherichia coli & $11(50 \%)$ & & \\
\hline Klebsiella Pneumoniae & $7(31.82 \%)$ & & \\
\hline Serratia marcescens & $2(9.09 \%)$ & & \\
\hline Enterobacter cloacae & $1(4.55 \%)$ & & \\
\hline
\end{tabular}

Data presented as mean \pm SEM or number (\%). Preterm newborns (Gestational age $<37$ weeks); Term newborns (Gestational age $>37$ weeks); Early-onset sepsis (EOS): onset of symptoms before 72 hours of life; Late-onset sepsis (LOS): onset of symptoms beyond 72 hours after birth and before 28 days; NVD: non-vaginal delivery; CS: caesarean section. 


\begin{tabular}{|lc|}
\hline Variable & Sepsis $(\mathbf{n = 6 1 )}$ Control $(\mathbf{n = 3 2 )}$ \\
\hline Enterobacter aerogenes & $\mathbf{P}(4.55 \%)$ \\
\hline $\begin{array}{l}\text { Data presented as mean } \pm \text { SEM or number (\%). Preterm newborns (Gestational age }<37 \text { weeks); Term newborns (Gestational age }>37 \text { weeks); Early-onset } \\
\text { sepsis (EOS): onset of symptoms before 72hours of life; Late-onset sepsis (LOS): onset of symptoms beyond } 72 \text { hours after birth and before 28 days; } \\
\text { NVD: non-vaginal delivery; CS: caesarean section. }\end{array}$ \\
\hline
\end{tabular}

\subsection{Expression of BTLA on mDC was higher in septic neonates compared with non-septic neonates}

The apoptotic loss and functional capacity of circulating DCs in sepsis patients are well known[10]. Given BTLA's role in attenuating DCs function, we looked for the differences of BTLA expression on mDCs (determined by CD11 $\mathrm{c}^{\text {high }}$ MHCI ${ }^{\text {high }}$ ) from the peripheral blood of septic $(n=61)$ and non-septic $(n=32)$ neonates patients to access the role of BTLA expression on $\mathrm{mDC}$ in neonatal sepsis. Our data showed that the percentage of $B T L A^{+} m D C s$ was significantly higher in septic neonates when compared to non-septic neonates (Fig. 1A-C). Our data also showed that the BTLA expression level in preterm neonates (gestational ages < 37 weeks), lower weight neonates (body weight $<2500 \mathrm{~g}$ ) and Apgar score at $1 \mathrm{~min}<9$ neonates were higher than that in full term, normal birth weight (body weight $>2500 \mathrm{~g}$ ) and Apgar score at 1 min $>9$ neonates (Fig. 1D). Hence, we wonder whether BTLA expression level on mDCs could be a predictive marker in neonatal sepsis. To verify our hypothesis, the ROC curve analysis was performed. Although the predictive effect was not as strong as PCT (Areas under the curve (AUCs) $0.9319,95 \% \mathrm{Cl}: 0.8840$ to 0.9797 ), the percentage of BTLA ${ }^{+} \mathrm{mDCs}$ still was a relatively better marker in predicting neonatal sepsis (AUCs $0.7113,95 \%$ Cl: 0.6004 to 0.8223 ) when compared with WBC (AUCs $0.5966,95 \%$ Cl:0.4839 to 0.7092) and neutrophil (AUCs $0.6726,95 \%$ Cl:0.5646 to 0.7807 ) (Fig. 1E).

\subsection{Percentage of circulating BTLA ${ }^{+}$mDCs was positively correlated with the severity of neonatal sepsis}

To assess the role of BTLA in neonates diagnosed with sepsis, septic neonate group was further divided into two sub-groups: severe neonatal sepsis (neonates died for sepsis or the condition of septic neonates worsen and needed to be transferred to superior hospitals, Table 2) and non-severe neonatal sepsis. Our data showed that the percentage of BTLA ${ }^{+} m D C$ s from severe septic neonates was higher than that from non-severe septic neonates (Fig. 2A). Later, we selected 5 of neonates in each group to study the changes of BTLA expression on mDCs along with prolonged hospitalization. Data demonstrated that the percentage of BTLA ${ }^{+} \mathrm{mDC}$ s was stepwise increased in severe septic neonates throughout the whole 10 days of hospitalization, which in non-severe septic neonates started to decrease from the 4th day (Fig. 2B). Furthermore, correlation analysis showed the percentage of circulating BTLA ${ }^{+} \mathrm{mDC}_{\mathrm{S}}$ was positively correlated with the duration of hospitalization in non-severe septic patients (Fig. 2C). However, similar correlation was not found in control group (Fig. 2D). All these data suggested higher expression of BTLA in mDCs may not conducive to the control of infection and evenwould lead to a pejorative outcome in neonates with sepsis. 
Table 2

The detail characteristics in patients with severe neonatal sepsis

\begin{tabular}{|c|c|c|c|c|c|c|c|c|c|c|c|c|}
\hline \multirow[t]{2}{*}{ No } & \multirow{2}{*}{$\begin{array}{l}\text { Gender } \\
(\mathrm{M} / \mathrm{F})\end{array}$} & \multirow{2}{*}{$\begin{array}{l}\text { Age } \\
\text { (days) }\end{array}$} & \multirow{2}{*}{$\begin{array}{l}\text { Gestational } \\
\text { age } \\
\text { (weeks) }\end{array}$} & \multirow{2}{*}{$\begin{array}{l}\text { Delivery } \\
\text { type } \\
\text { (NVD/CS) }\end{array}$} & \multirow{2}{*}{$\begin{array}{l}\text { Birth } \\
\text { weight } \\
(\mathrm{kg})\end{array}$} & \multicolumn{3}{|c|}{ Apgar score } & \multirow{2}{*}{$\begin{array}{l}\text { Site of } \\
\text { infection }\end{array}$} & \multicolumn{2}{|c|}{ Microbiological diagnosis } & \multirow{2}{*}{$\begin{array}{l}\text { Durati } \\
\text { hospit } \\
\text { (days) }\end{array}$} \\
\hline & & & & & & $1 \mathrm{~min}$ & $5 \mathrm{~min}$ & $10 \mathrm{~min}$ & & $\begin{array}{l}\text { Bacterial } \\
\text { culture }\end{array}$ & Bacterial name & \\
\hline 1 & $\mathrm{~F}$ & 4 & 38 & NVD & 3.53 & 10 & 10 & 10 & No found & Negative & & 1 \\
\hline 2 & $\mathrm{~F}$ & 16 & 38 & NVD & 3.12 & 10 & 10 & 10 & Blood & Positive & Klebsiella Pneumoniae & 4 \\
\hline 3 & $\mathrm{~F}$ & 8 & 35 & NVD & 2.28 & 10 & 10 & 10 & Abdominal & Negative & & 9 \\
\hline 4 & M & 9 & 39 & CS & 3.36 & 10 & 10 & 10 & Brain & Positive & Escherichia coli & 6 \\
\hline 5 & M & 27 & 31 & NVD & 1.1 & 7 & 9 & 10 & Lung & Positive & Serratia marcescens & 47 \\
\hline 6 & M & 16 & 39 & CS & 3.49 & 10 & 10 & 10 & Skin & Positive & Staphylococcus aureus & 2 \\
\hline 7 & M & 15 & 38 & NVD & 4.05 & 10 & 10 & 10 & Blood & Positive & Escherichia coli & 3 \\
\hline 8 & $\mathrm{~F}$ & 1 & 32 & NVD & 1.66 & 8 & 9 & 9 & No found & Negative & & 12 \\
\hline 9 & M & 1 & 28 & NVD & 1.32 & 5 & 7 & 8 & No found & Negative & & 15 \\
\hline 10 & $\mathrm{~F}$ & 3 & 41 & CS & 3.3 & 8 & 10 & 10 & Blood & Positive & Group B streptococcus & 2 \\
\hline 11 & M & 22 & 38 & NVD & 2.6 & 10 & 10 & 10 & $\begin{array}{l}\text { Abdominal } \\
\text { and Brain }\end{array}$ & Negative & & 11 \\
\hline 12 & M & 1 & 37 & CS & 3 & 10 & 10 & 10 & Blood & Positive & Pediococcus pentosaceus & 2 \\
\hline 13 & M & 1 & 30 & NVD & 1.35 & 2 & 5 & 6 & No found & Negative & & 1 \\
\hline 14 & M & 1 & 38 & NVD & 2.52 & 10 & 10 & 10 & No found & Negative & & 15 \\
\hline
\end{tabular}

\subsection{BTLA expression associated with a lower phagocytosis ability and bactericidal capacity of mDCs}

As phagocytosis is one of the important functions for APC to mediate innate immunity and antigen processing, we measured the phagocytosis ability of $B_{T L A^{+} m D C s}$ to access the regulation effect of BTLA on the cell function of $m D C s$. BTLA ${ }^{+} m D C s$ or $B T L A^{-} m D C s$ from neonatal sepsis patients were sorted by flow cytometry, and incubated with FITC-labeled dextran. The phagocytosis capacity of BTLA ${ }^{+} m D C s$ or BTLA ${ }^{-}$mDCs was measured by flow cytometry. Comparative analysis indicated that BTLA ${ }^{+} \mathrm{mDC}$ s exhibited lower FITC-dextran uptake capacity, whereas BTLA ${ }^{-}$mDCs uptake more dextran, suggesting BTLAexpressing mDCs appeared with lower phagocytosis (Fig. 3A\&B). In addition, we also designed bactericidal assay to further measure the regulatory effect of

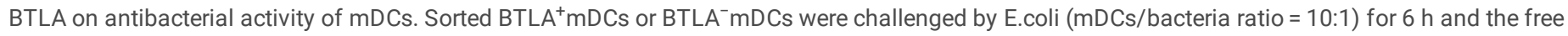
bacteria from each group infection system were grown in $\mathrm{MH}$ agar to access the bactericidal capacity of different group of mDCs. As expected, more $\mathrm{CFU}$ number was found in $\mathrm{BTLA}^{+} \mathrm{mDC}$ group when compared with that in BTLA-mDCs group, indicating BTLA expression in mDCs suppressed the antibacterial capacity of mDCs (Fig. 3C\&D).

\subsection{BTLA negatively regulated the synthesis of HLA-DR in mDCs}

After internalizing exogenous pathogen, DCs were activated and mobilized peptide-MHC-II complexes to the surface and increase their stability to improve recognition by $\mathrm{CD}^{+} \mathrm{T}$ cells and induce adaptive immune responses. Therefore, the antigen-presenting function of DCs dependent on the peptide-MHC-II complexes expressed on the surface of DCs to a large extent. HLA-DR, an antigen presenting molecule, is one of the most powerful allogenic stimulator corresponding to MHC class-II complexes expressed on DCs. Here, we detected the expression of HLA-DR in mDCs from neonatal patients and analyzed the correlation of BTLA and HLA-DR expression in mDCs to verify the regulation effect of BTLA on mDCs function. Flow cytometry analysis demonstrated that the expression level of HLA-DR in mDCs from septic neonates was lower than that from non-septic neonates (Fig. 4A). Besides, a significantly lower expression level of HLA-DR in mDCs from neonates with severe sepsis was found when compared with non-severe septic neonates (Fig. 4B). Moreover, in non-severe septic neonates, the expression of HLA-DR in mDCs showed an upward trend after the 4th days of hospitalization, whereas the expression in neonates with severe sepsis had no obvious change throughout the whole hospitalization (Fig. 4C). Correlation analysis demonstrated that the expression of HLA-DR in mDCs was negatively correlated with the percentage of BTLA ${ }^{+} m D C$ s in septic neonates (Fig. 4D). However, similar negative correlation could not be found in control group (Fig. 4E). Collectively, a higher level of BTLA in mDCs may affect the synthesis of HLA-DR, which leads the antigen-presenting function of mDCs to be impaired.

\subsection{BTLA expression in mDCs contributes to decreasing pro-inflammatory response but rising IL-10 levels during the infection of E.coli}


Publication has reported both splenic and lymph node DCs during sepsis fail to acquire a mature state (expression of CD80, CD86, and CD40) and thus release a lower quantity of IL-12 but higher quantity of IL-10, which resulting unresponsiveness and/or tolerance of T cells [24]. In our study, the supernatant expression of TNF-a, IL-12 and IL-10 in sorted BTLA ${ }^{+}$DDCs and BTLA ${ }^{-} m D C s$ from septic neonates was measured after infected by E.coli for 6 h. Data showed that BTLA ${ }^{+} m D C$ s secreted a lower level of TNF-a (Fig. 5A) and IL-12(Fig. 5B) but higher level of IL-10 (Fig. 5C) when compared with BTLA ${ }^{-} \mathrm{mDCs}$, indicating a role for BTLA in shifting $\mathrm{mDC}$ from a pro-inflammatory phenotype toward an anti-inflammatory phenotype and the persistent anti-inflammatory response is believed to contribute to the profound state of immune paralysis and late septic death $[25,26]$.

\section{Discussion}

Neonatal sepsis is the constellation of symptoms occurring an infection with bacterial, viral, or fungal (mostly yeast) microorganisms that leads to a systemic inflammatory response in neonates [27, 28]. Despite the extensive use of broad-spectrum antibiotics, ventilator management, resuscitative strategies, and improvement of nutritional support, four of every 10 neonates that develop sepsis die or experience major disability [29]. The newborn immune system is complicated by its immaturity, however, akin to adult counterparts, immune exhaustion and dysfunction also occurred in neonates[30]. Published studies have shown that co-inhibitory receptors, including PD-1, CTLA-4 and BTLA, are contributed to the progression of sepsis [20,31-34]. Although these receptors were originally thought primarily to be inducers of anergy in lymphocytes, such as $C D 4^{+} \mathrm{T}$ cells, increasing evidence verified that innate cell populations, including macrophages, monocytes and DCs, also appear to be induced to express co-inhibitory receptors, including BTLA, and the ligation of these receptors may have effects on them as well[20]. BTLA has been showed to be detrimental in the bacteria clearance in mice by impairing the recruitment of innate inflammatory immune cells to the infected sites as well as inhibiting them to present antigen to adaptive immune system [20]. However, the expression of BTLA as well as its regulation effect on DCs in neonates with sepsis remains to be elucidated.

In our study, we found the percentage of BTLA ${ }^{+}$mDCs in septic neonates was significantly higher than that in non-septic neonates. We conducted ROC curve analysis, and the percentage of BTLA ${ }^{+} m D C s$ was a relatively better marker in predicting neonatal sepsis even though the predictive effect was not as strong as PCT. Yang and his colleagues confirmed that BTLA is selectively expressed in CD8 $a^{+} D C s$, but not CD8 $a^{-}$DCs to regulate the bacteria expansion in mice [35]. But we did not further determine which subtypes of mDCs were elevated during neonatal sepsis, which would be our next study point. Complex immune reactions were developed during sepsis, which can be conceptualized as an occurrence of a pro-inflammatory along with a concomitant anti-inflammatory response and this persistent anti-inflammatory response is believed to contribute to the profound state of immune paralysis and late morbid outcome [26, 36]. Therefore, the increased expression of inhibitory molecular in the early stage of sepsis could prevent the cytokine storm from inducing organ failure [37]. In this regard, BTLA has been shown could directly inhibit LPS responses in DCs and M $\phi s$, and the agonistic agents of BTLA might have therapeutic potential for LPS-induced endotoxic shock, which an overwhelming and uncontrolled immune responses would occur in the early phase of infection [37]. However, if their expression still keeps in a high level during the immunosuppression stage, $T$ cell anergy or immune cell deactivation would be induced and that is detrimental for the bacteria clearance. Our data provided herein demonstrated that those neonates with severe sepsis showed higher level of BTLA ${ }^{+} \mathrm{mDCs}_{\mathrm{s}}$ than non-severe sepsis. As the extension of the days of hospitalization, the percentage of BTLA ${ }^{+} m D C$ s showed a stepwise increase in severe septic neonates, whereas it began to decrease from the 4 th days of hospitalization in non-severe septic neonates. We feel that our findings provided here suggested that not only did the percentage of BTLA ${ }^{+} m D C$ s correlate to the severity of sepsis, but also it was valuable for prognostic evaluation of sepsis. The increased level of BTLA ${ }^{+} m D C s$ is not conducive to the control of infection, in other words, the higher level of BTLA ${ }^{+} \mathrm{mDC}$ in the late stage of sepsis would be a predictor of pejorative outcome in neonates with sepsis.

BTLA has been confirmed to be detrimental for the clearance of bacteria, including L. monocytogenes and Plasmodium, suggesting a role for in inhibiting innate phagocytic cell action $[19,35,38]$. Prior study also demonstrated that BTLA-expressing CD11 ${ }^{+}$APCs from tuberculosis infected patient exhibit lower antigen uptake capacity than BTLA negative CD11c APCs [21]. Consistently, our data demonstrated that BTLA ${ }^{+}$mDCs from septic neonates exhibited lower phagocytosis ability and bactericidal capacity when compared with BTLA ${ }^{-} m D C s$. In addition to impairing the antigen-uptake capacity of DCs, the occurrence of BTLA also has been found could negatively regulate the antigen-presenting capacity of innate cells to adaptive immune system, which is another disadvantage for bacteria clearance. CD8 $\mathrm{a}^{+} \mathrm{DC}$ s from BTLA deficient mice are less efficient at cross-presenting Listerial antigen to CD $8^{+} \mathrm{T}$ cells resulting in less ability to induce T cells proliferation and IFN-y production [35]. Our previous study also found that BTLA-expressing CD11 ${ }^{+}$APCs from tuberculosis infected patients display lower stimulatory capacity of $C D 3^{+} \mathrm{T}$ cells, especially $\mathrm{CD} 8^{+} \mathrm{T}$ cells, because of the diminished expression of HLA-DR and lower production of IL-6[21]. Interestingly, in this study, we found HLA-DR expression in mDCs was lower in neonates with sepsis than neonates with no sepsis. However, a much higher level expression of HLA-DR in mDCs was found in septic neonates than severe septic neonates and an increasing tendency was found as the extension of hospitalization, which keeps in a relative stable expression in severe septic neonates. Furthermore, HLA-DR expression in mDCs was negatively regulated by BTLA in neonates with sepsis but not no-sepsis. Combined with previous studies, our findings provided new evidence for the regulation effect of BTLA in the cell function of mDCs and might interpret partially why neonates with higher levels of BTLA in mDCs display severe sepsis and longer duration of hospitalization.

Cytokine plays an important role in influencing the cell function of DCs. It is verified that co-inhibitory receptors, including PD-1 and BTLA, could not only inhibiting innate cells effector function but also shifting them into anti-inflammatory phenotypes as well. The induced or secreted anti-inflammatory mediators, like IL-10, are thought to cause innate immune cells, including monocytes, macrophages and DCs, to become dysfunctional. The dysfunction includes reduced IL-1, TNF-a, and IL-6 pro-inflammatory cytokine, but increased IL-10, which are not conducive to the control of infection during sepsis[36]. As an activator of DCs, the expression of IL-12 also was found to be reduced during sepsis[39, 40].In our study, we found BTLA-expressing mDCs from septic neonates indeed secreted lower levels of TNF- $\alpha$ and IL-12, but higher IL-10 after infected by E.coli. However, study with acute experimental sepsis induction in mice model demonstrated that IL-10, TNF- $a$, IFN- $y$ and IL- 6 all were increased, whereas only IL-10 expression was reduced when diminished BTLA expression[20]. In other words, BTLA expression only contributes to the regulation of anti-inflammatory system but not pro-inflammatory system. There are 
potentially many reasons for why the different results occurred. Firstly, the sample collection time in mice could be chose, like that study was in $24 \mathrm{~h}$ post-CLP; however, it was not possible to establish the exact time-point that the patient's septic insult and pathogen challenge began. Also, the nature and source of the initial nidus of infection in those neonates included into our study were differed. Therefore, BTLA still could be an important regulatory protein on mDCs. However, further studies should be proceeded to investigate the potential molecular mechanism of how BTLA regulate the mDCs function to regulate the sepsis outcome in neonates.

\section{Conclusions}

Our study firstly provided evidence that BTLA-expressing mDCs were elevated in neonates with sepsis and the expression level in mDCs was positively correlated to the severity of sepsis. In addition, our findings suggested that BTLA negatively regulated the phagocytosis capacity and bactericidal ability of mDCs and lower their antigen-presenting ability by reducing the expression of HLA-DR as well as altered cells into an anti-inflammatory phenotype. Therefore, BTLA expression in mDCs could be useful predictive marker for neonatal sepsis and targeting BTLA expression in mDCs may be a new therapeutic strategy.

\section{Abbreviations}

BTLA:B and T lymphocyte attenuator; mDCs:Melyoid dendritic cells; Tregs:Regulatory T cells; CTLA-4:Cytotoxic T-lymphocyte antigen-4; MFI:Mean fluorescence intensity; APCs:Antigen-presenting cells; HLA-DR:Human leukocyte antigen-DR; PD-1:Programmed death receptor-1; CLP:Cecal ligation and puncture;

\section{Declarations}

\section{Ethical approval and consent to participate}

The study was approved by the Internal Review and the Ethics Boards of Affiliated Xiaolan Hospital, Southern Medical University and Children's Hospital Affiliated to Zhengzhou University and informed consent was obtained from all participants.

\section{Availability of data and materials}

All data generated or analysed during this study are included in this published article.

\section{Availability of supporting data}

Not applicable

\section{Consent for publication}

Not applicable

\section{Competing interests}

The authors declare that they have no competing interests.

\section{Funding}

This study was supported by the Science and Technology Project of Zhongshan 2015B1076 (to G-HC), the Science and Technology Project of Zhongshan 2019B1056 (to W-DW), the Medical Research Funding of Guangdong A2019286 (to W-DW) and the Health Project of Zhongshan $2019 \mathrm{~J} 274$ (to X-RY).

\section{Authors' contributions}

W-DW and G-HC conducted the general study concepts and design and wrote the manuscript. All of the experiments were performed by X-RY and M-FG. Z-FP, M-JQ, J-YW, Y-LL, W-TD and JJ substantially contributed to the design and conception of the human studies. All authors have given final approval for this manuscript to be published.

\section{Acknowledgments}

We thank the selected nurses, doctors and staffs at the Department of Clinical medicine laboratory, Affiliated Xiaolan Hospital, Southern Medical University, China, and Department of Clinical Medicine Laboratory, Children's Hospital Affiliated to Zhengzhou University, China for collection of study subjects and clinical data.

\section{References}

1. Qazi SA, Stoll BJ: Neonatal sepsis: a major global public health challenge. Pediatr Infect Dis J 2009, 28(1 Suppl):S1-2.

2. You D, Hug L, Ejdemyr S, Idele P, Hogan D, Mathers C, Gerland P, New JR, Alkema L, United Nations Inter-agency Group for Child Mortality E: Global, regional, and national levels and trends in under-5 mortality between 1990 and 2015, with scenario-based projections to 2030: a systematic analysis by the UN Inter-agency Group for Child Mortality Estimation. Lancet 2015, 386(10010):2275-2286.

3. Liu L, Oza S, Hogan D, Perin J, Rudan I, Lawn JE, Cousens S, Mathers C, Black RE: Global, regional, and national causes of child mortality in 2000-13, with projections to inform post-2015 priorities: an updated systematic analysis. Lancet 2015, 385(9966):430-440. 
4. Chao YH, Wu HP, Wu KH, Tsai YG, Peng CT, Lin KC, Chao WR, Lee MS, Fu YC: An increase in CD3+CD4+CD25+ regulatory T cells after administration of umbilical cord-derived mesenchymal stem cells during sepsis. PLOS One 2014, 9(10):e110338.

5. Skirecki T, Borkowska-Zielinska U, Zlotorowicz M, Hoser G: Sepsis immunopathology: perspectives of monitoring and modulation of the immune disturbances. Arch Immunol Ther Exp (Warsz) 2012, 60(2):123-135.

6. Hotchkiss RS, Monneret G, Payen D: Immunosuppression in sepsis: a novel understanding of the disorder and a new therapeutic approach. Lancet Infect Dis 2013, 13(3):260-268.

7. Ding Y, Chung CS, Newton S, Chen Y, Carlton S, Albina JE, Ayala A: Polymicrobial sepsis induces divergent effects on splenic and peritoneal dendritic cell function in mice. Shock 2004, 22(2):137-144.

8. Efron PA, Martins A, Minnich D, Tinsley K, Ungaro R, Bahjat FR, Hotchkiss R, Clare-Salzler M, Moldawer LL: Characterization of the systemic loss of dendritic cells in murine lymph nodes during polymicrobial sepsis. J Immuno/ 2004, 173(5):3035-3043.

9. Tinsley KW, Grayson MH, Swanson PE, Drewry AM, Chang KC, Karl IE, Hotchkiss RS: Sepsis induces apoptosis and profound depletion of splenic interdigitating and follicular dendritic cells. J Immunol 2003, 171(2):909-914.

10. Kumar V: Dendritic cells in sepsis: Potential immunoregulatory cells with therapeutic potential. Mol Immuno/ 2018, 101:615-626.

11. Jones A, Bourque J, Kuehm L, Opejin A, Teague RM, Gross C, Hawiger D: Immunomodulatory Functions of BTLA and HVEM Govern Induction of Extrathymic Regulatory T Cells and Tolerance by Dendritic Cells. Immunity 2016, 45(5):1066-1077.

12. Li S, Zhang M, Xiang F, Zhao J, Jiang C, Zhu J: Dendritic cells expressing BTLA induces CD8+ T cell tolerance and attenuates the severity of diabetes. Vaccine 2011, 29(44):7747-7751.

13. Watanabe N, Gavrieli M, Sedy JR, Yang J, Fallarino F, Loftin SK, Hurchla MA, Zimmerman N, Sim J, Zang X et al: BTLA is a lymphocyte inhibitory receptor with similarities to CTLA-4 and PD-1. Nat Immunol 2003, 4(7):670-679.

14. Murphy TL, Murphy KM: Slow down and survive: Enigmatic immunoregulation by BTLA and HVEM. Annu Rev Immuno/ 2010, 28:389-411.

15. Sedy JR, Gavrieli M, Potter KG, Hurchla MA, Lindsley RC, Hildner K, Scheu S, Pfeffer K, Ware CF, Murphy TL et al: B and T lymphocyte attenuator regulates T cell activation through interaction with herpesvirus entry mediator. Nat Immunol 2005, 6(1):90-98.

16. Miller ML, Sun Y, Fu YX: Cutting edge: B and T lymphocyte attenuator signaling on NKT cells inhibits cytokine release and tissue injury in early immune responses. J Immunol 2009, 183(1):32-36.

17. Wang Y, Subudhi SK, Anders RA, Lo J, Sun Y, Blink S, Wang Y, Wang J, Liu X, Mink K et al: The role of herpesvirus entry mediator as a negative regulator of T cell-mediated responses. J Clin Invest 2005, 115(3):711-717.

18. Krieg C, Boyman O, Fu YX, Kaye J: B and T lymphocyte attenuator regulates CD8+ T cell-intrinsic homeostasis and memory cell generation. Nat Immunol 2007, 8(2):162-171.

19. Sun Y, Brown NK, Ruddy MJ, Miller ML, Lee Y, Wang Y, Murphy KM, Pfeffer K, Chen L, Kaye $J$ et al: B and T lymphocyte attenuator tempers early infection immunity. J Immunol 2009, 183(3):1946-1951.

20. Shubin NJ, Chung CS, Heffernan DS, Irwin LR, Monaghan SF, Ayala A: BTLA expression contributes to septic morbidity and mortality by inducing innate inflammatory cell dysfunction. J Leukoc Bio/ 2012, 92(3):593-603.

21. Wang WD, Gao YC, Lu YB, Zhang JA, Liu GB, Kong B, Xiang WY, Dai YC, Yu SY, Jia Y et al: BTLA-expressing CD11c antigen presenting cells in patients with active tuberculosis exhibit low capacity to stimulate T cell proliferation. Cell Immunol 2017, 311:28-35.

22. Cai X, Ge N, Rong R, Lu Y, Zhang J, Xu J: High expression of BTLA and B7-H4 on the surface of myeloid dendritic cells has a negative regulatory effect on their anti-tuberculosis immunity activity in pleural tuberculosis patients. Tuberculosis (Edinb) 2019, 119:101877.

23. Subspecialty Group of Neonatology Pediatric Society Chinese Medical A, Editorial Board Chinese Journal of P: [Protocol for diagnosis and treatment of neonatal septicemia]. Zhonghua Er Ke Za Zhi 2003, 41(12):897-899.

24. Flohe SB, Agrawal H, Schmitz D, Gertz M, Flohe S, Schade FU: Dendritic cells during polymicrobial sepsis rapidly mature but fail to initiate a protective Th1-type immune response. J Leukoc Biol 2006, 79(3):473-481.

25. Hotchkiss RS, Nicholson DW: Apoptosis and caspases regulate death and inflammation in sepsis. Nat Rev Immuno/ 2006, 6(11):813-822.

26. Hotchkiss RS, Monneret G, Payen D: Sepsis-induced immunosuppression: from cellular dysfunctions to immunotherapy. Nat Rev Immunol 2013, 13(12):862-874.

27. Shane AL, Sanchez PJ, Stoll BJ: Neonatal sepsis. Lancet 2017, 390(10104):1770-1780.

28. Kaukonen KM, Bailey M, Pilcher D, Cooper DJ, Bellomo R: Systemic inflammatory response syndrome criteria in defining severe sepsis. N Engl $J$ Med 2015, 372(17):1629-1638.

29. Group IC, Brocklehurst P, Farrell B, King A, Juszczak E, Darlow B, Haque K, Salt A, Stenson B, Tarnow-Mordi W: Treatment of neonatal sepsis with intravenous immune globulin. N Engl J Med 2011, 365(13):1201-1211.

30. Haeney M: Infection determinants at extremes of age. J Antimicrob Chemother 1994, 34 Suppl A:1-9.

31. Young WA, Fallon EA, Heffernan DS, Efron PA, Cioffi WG, Ayala A: Improved survival after induction of sepsis by cecal slurry in PD-1 knockout murine neonates. Surgery 2017, 161(5):1387-1393.

32. Brahmamdam P, Inoue S, Unsinger J, Chang KC, McDunn JE, Hotchkiss RS: Delayed administration of anti-PD-1 antibody reverses immune dysfunction and improves survival during sepsis. J Leukoc Biol 2010, 88(2):233-240.

33. Inoue S, Bo L, Bian J, Unsinger J, Chang K, Hotchkiss RS: Dosedependent effect of anti-CTLA-4 on survival in sepsis. Shock 2011, 36(1):38-44. 
34. Huang X, Venet F, Wang YL, Lepape A, Yuan Z, Chen Y, Swan R, Kherouf H, Monneret G, Chung CS et al: PD-1 expression by macrophages plays a pathologic role in altering microbial clearance and the innate inflammatory response to sepsis. Proc Natl Acad Sci U S A 2009, 106(15):6303-6308.

35. Yang X, Zhang X, Sun Y, Tu T, Fu ML, Miller M, Fu YX: A BTLA-mediated bait and switch strategy permits Listeria expansion in CD8alpha(+) DCs to promote long-term T cell responses. Cell Host Microbe 2014, 16(1):68-80.

36. Ng PC: Diagnostic markers of infection in neonates. Archives of Disease in Childhood - Fetal and Neonatal Edition 2004, 89:F229-F235.

37. Kobayashi Y, Iwata A, Suzuki K, Suto A, Kawashima S, Saito Y, Owada T, Kobayashi M, Watanabe N, Nakajima H: B and T lymphocyte attenuator inhibits LPS-induced endotoxic shock by suppressing Toll-like receptor 4 signaling in innate immune cells. Proc Natl Acad Sci U S A 2013, 110(13):5121-5126.

38. Adler G, Steeg C, Pfeffer K, Murphy TL, Murphy KM, Langhorne J, Jacobs T: B and T lymphocyte attenuator restricts the protective immune response against experimental malaria. $J$ Immuno/ 2011, 187(10):5310-5319.

39. Cheng T, Bai J, Chung CS, Chen Y, Biron BM, Ayala A: Enhanced Innate Inflammation Induced by Anti-BTLA Antibody in Dual Insult Model of Hemorrhagic Shock/Sepsis. Shock 2016, 45(1):40-49.

40. Lavoie PM, Huang Q, Jolette E, Whalen M, Nuyt AM, Audibert F, Speert DP, Lacaze-Masmonteil T, Soudeyns H, Kollmann TR: Profound lack of interleukin (IL)-12/IL-23p40 in neonates born early in gestation is associated with an increased risk of sepsis. J Infect Dis 2010, 202(11):1754-1763.

\section{Figures}

A

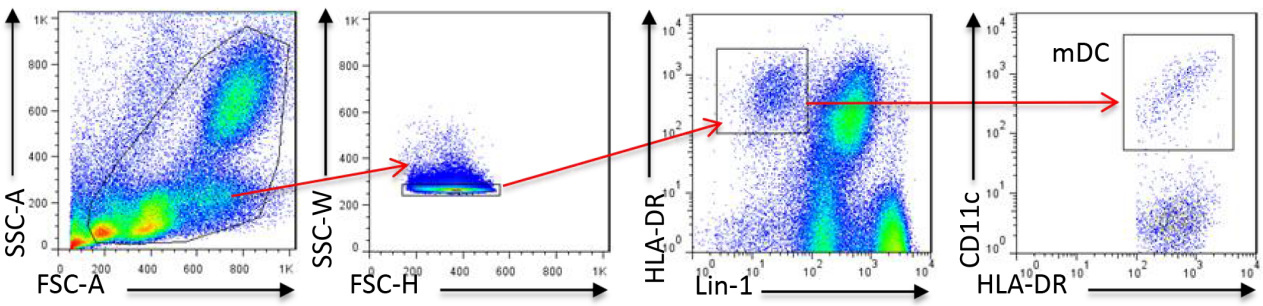

B
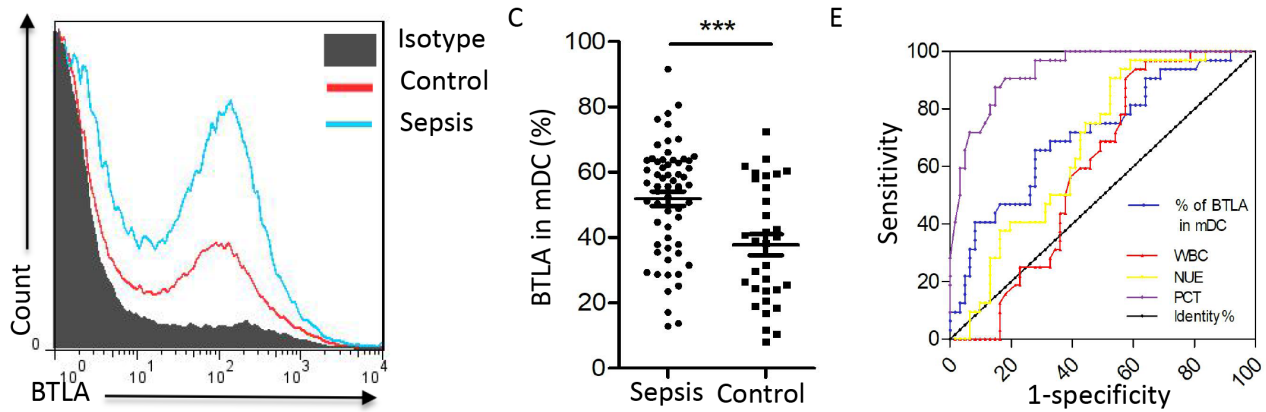

D

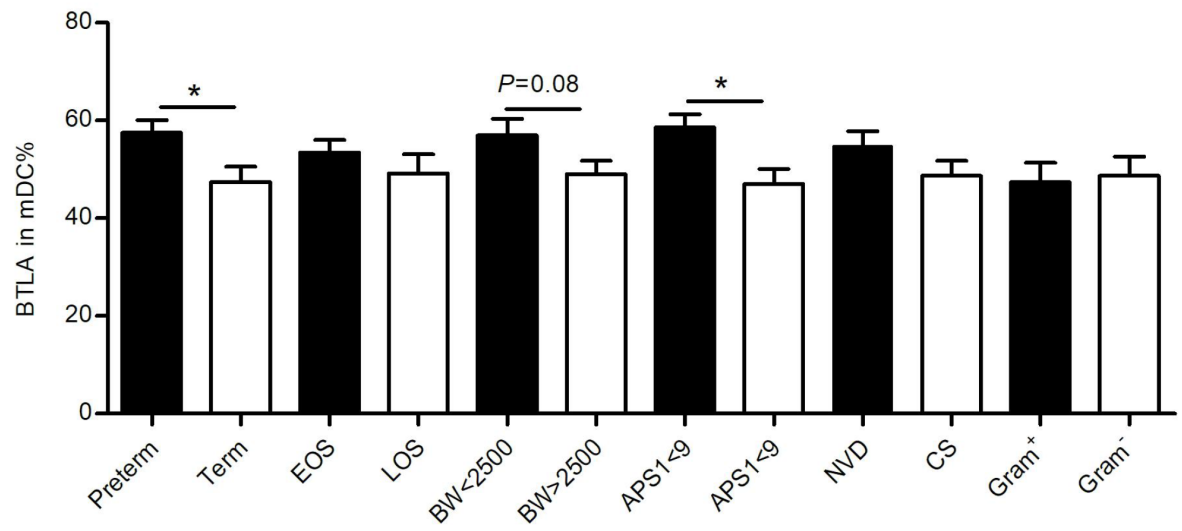

Figure 1

Figure 1 High percentage of BTLA+mDCs associated with the neonatal sepsis. Gating strategy of mDCs (CD11c+HLA-DR+DCs) and representative flow plots for BTLA expression by $\mathrm{mDCs}(\mathrm{A} \& \mathrm{~B})$. Histograms showing the percentage of BTLA+mDCs in sepsis and control group patients (C). Histograms showing the percentage of BTLA+mDCs between preterm and term, EOS and LOS, BW<2500g and BW>2500g, ASP1 $<9$ and ASP1 $>9$, NVD and CS, Gram + and Gramneonatal sepsis patients (D). Receiver Operating Characteristic (ROC) curve of the sensitivity and specificity of percentage of BTLA+mDCs (blue line), WBC (red line), neutrophil (yellow line) and PCT (pink line) (E). 
A

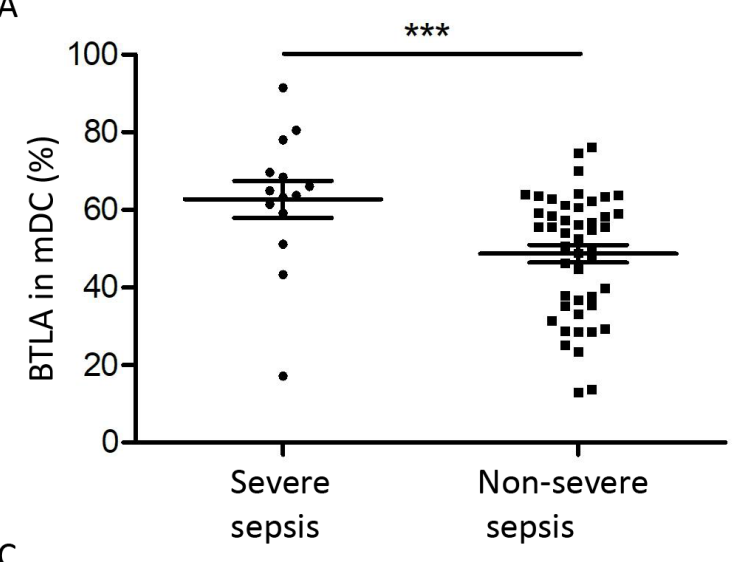

C

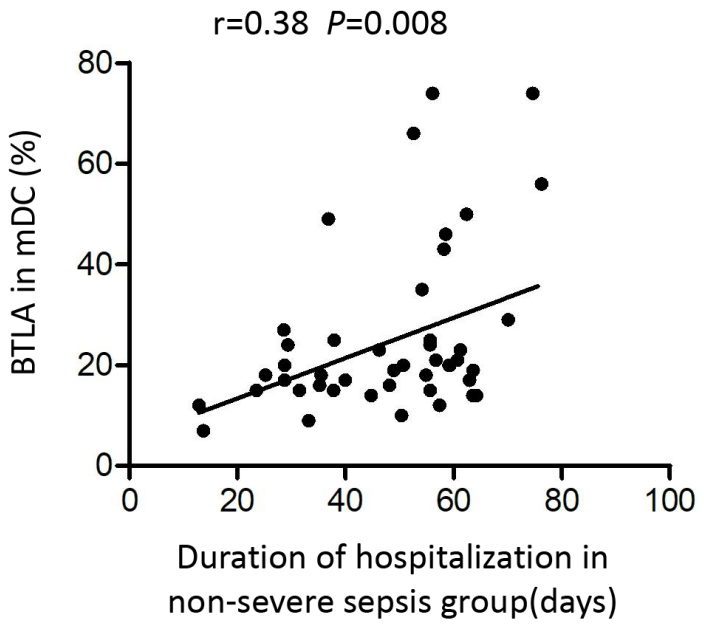

B

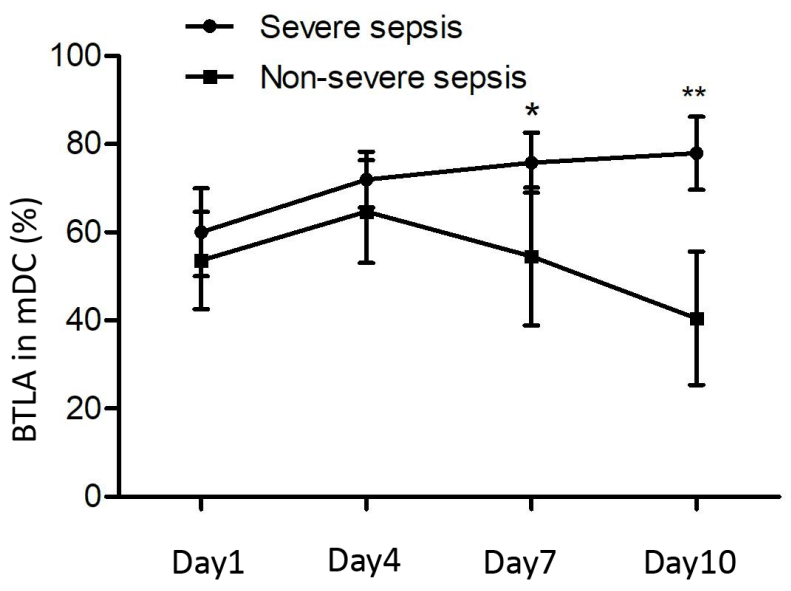

D

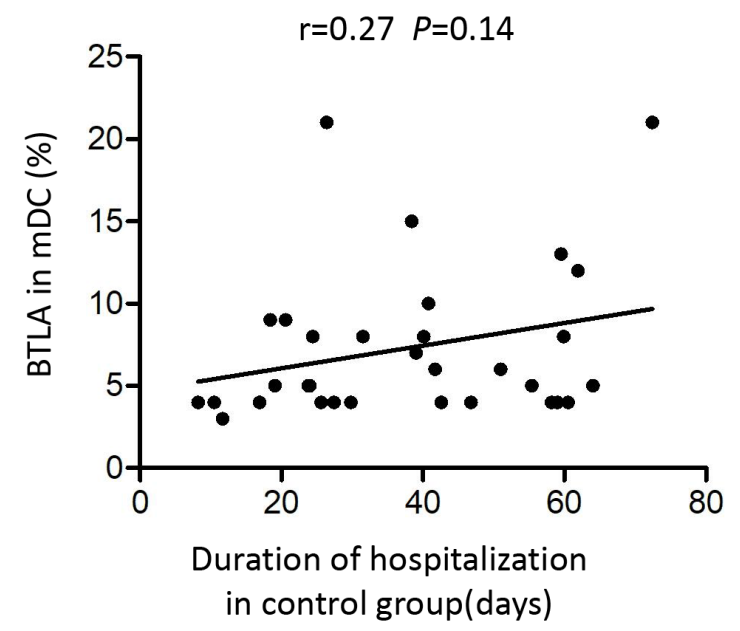

Figure 2

Expression level of BTLA on mDCs associated with the neonatal septic severity. Comparsion of BTLA+mDCs percentage within severe-septic ( $\mathrm{n}=14)$ and nonsevere septic neonates(A). Percentage of BTLA+mDCsin selected severe septic neonates $(n=5)$ and non-severe septic neonates ( $n=5)$ on the 1 st, 4 th, 7 th and 10th days of hospitalization (B).Correlation analysis between the percentage of BTLA+mDCs and the hospital length of stay in non-severe neonatal septic patients $(n=47)(C)$ or control group of neonates $(n=32)(D)$. 
A

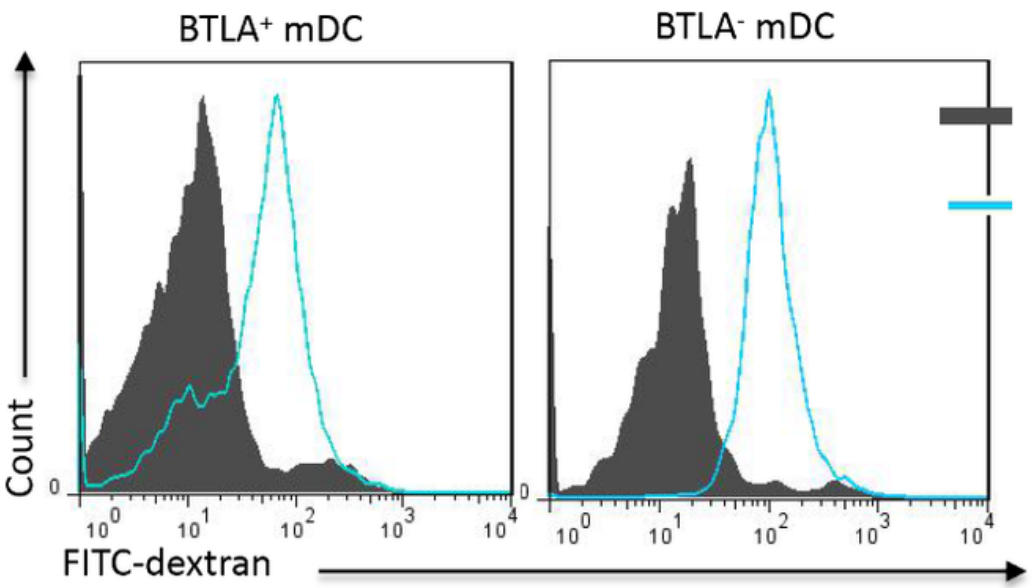

$\mathrm{C}$

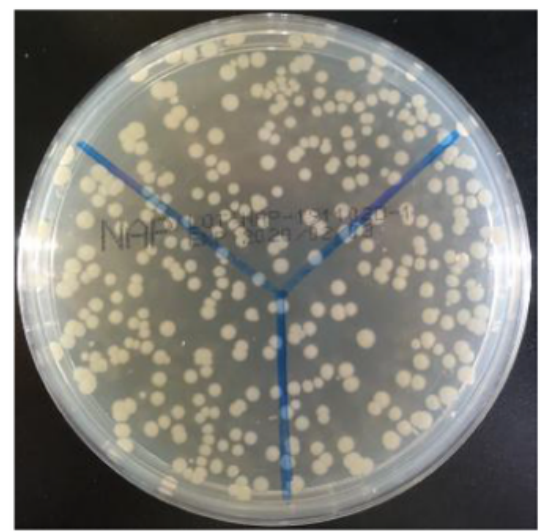

$\mathrm{BTLA}^{+} \mathrm{mDC}$

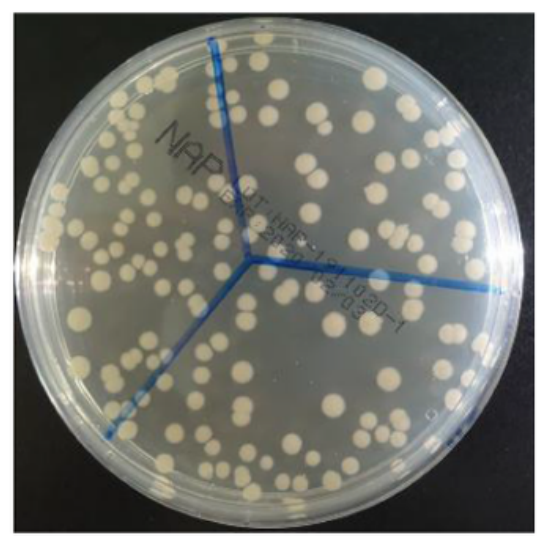

BTLA-mDC

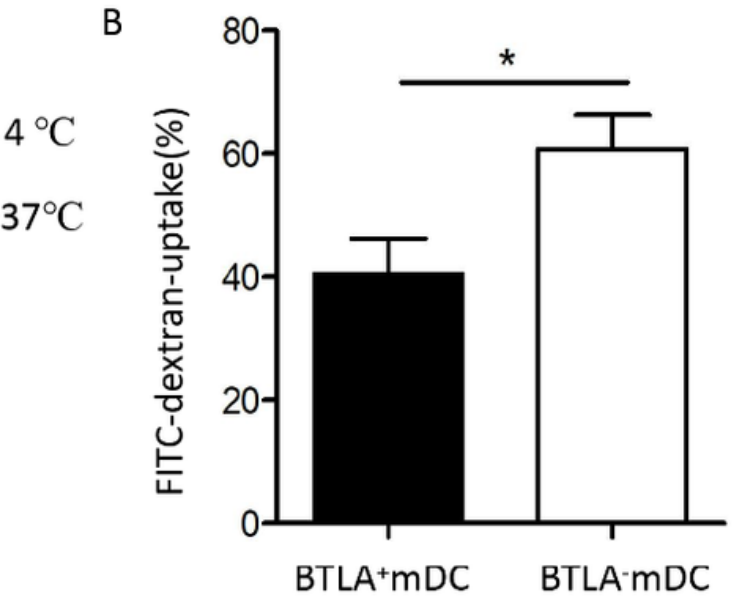

D

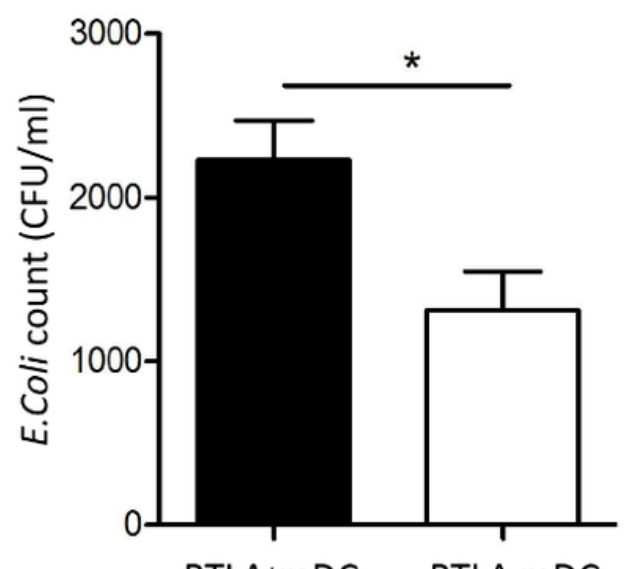

$\mathrm{BTLA}^{+} \mathrm{mDC}$

BTLA-mDC

\section{Figure 3}

BTLA+mDCs exhibited lower phagocytosis ability and bactericidal capacity. The FITC-dextran uptake assay was setup by incubating BTLA+mDCs and BTLAmDCs with FITC-dextran at $37^{\circ} \mathrm{C}$ and $4^{\circ} \mathrm{C}$ to compare the phagocytosis ability of BTLA+mDCs and BTLA-mDCs by analyzed with flow cytometry. Representative flow plots and histograms showing the phagocytosis ability of BTLA+mDCs and BTLA-mDCs(A\&B).BTLA+mDCs and BTLA-mDCsfrom neonatal septic patients were infected with E.coli for $6 \mathrm{~h}$ and the left free bacteria were put into $\mathrm{MH}$ agar in triplicate to determine bactericidal capacity of BTLA+mDCs and BTLA-mDCs. Representative bacteria colony pictures and histograms showing the number of colony-forming unit (CFU) in each group (C\&D). 

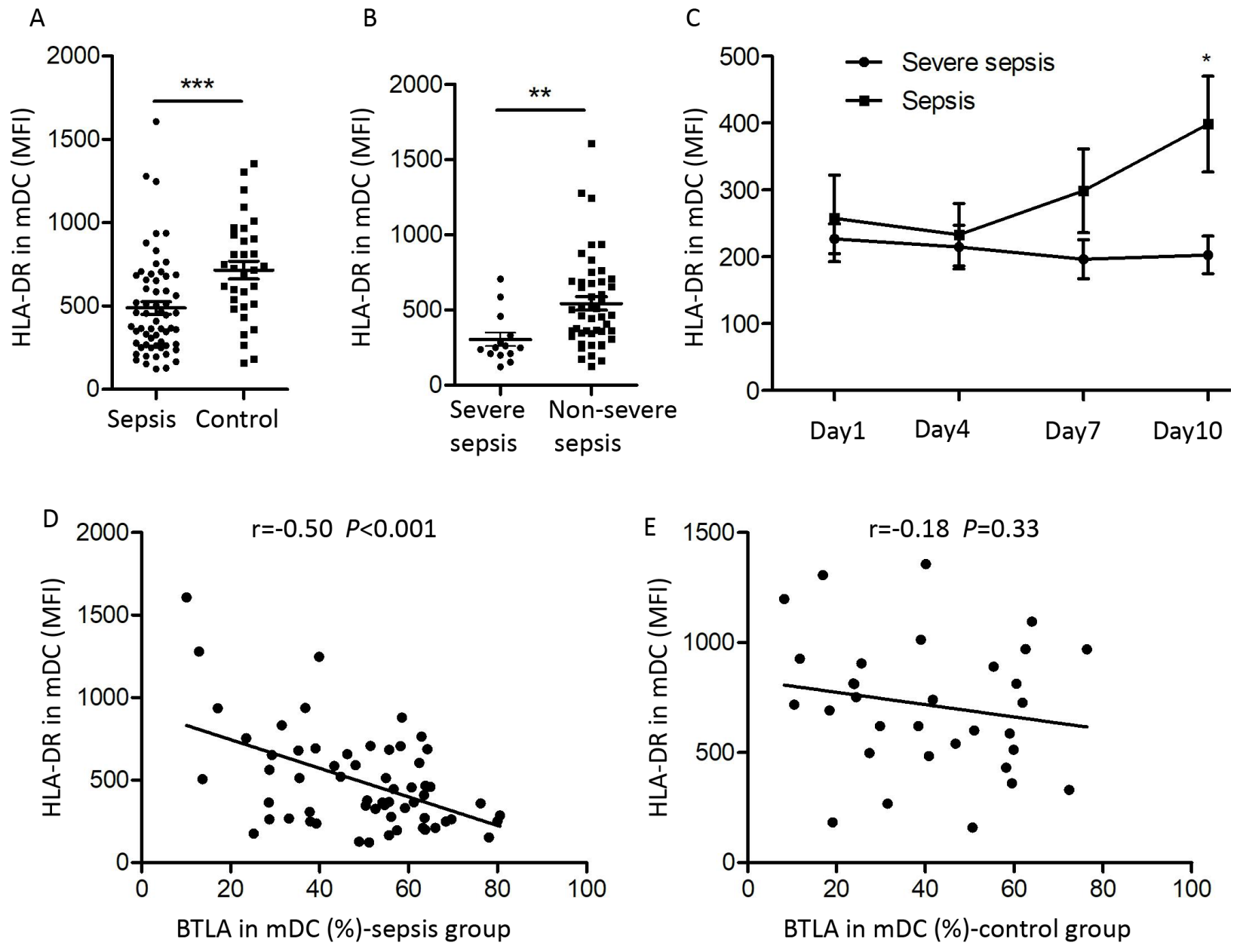

Figure 4

Expression of BTLA was negatively correlated with the HLA-DR expression on mDCs. Comparing the mean fluorescence intensity (MFI) of HLA-DR in mDCs between septic and control group neonatal patients (A). Histograms showing the MFI of HLA-DR in mDCs from neonates with severe sepsis or non-severe sepsis (B). Flow cytometer analysis of the expression of HLA-DR in mDCs from selected severe septic neonatal patients ( $n=5)$ and non-severe septic neonatal patients $(n=5)$ on the 1st, 4th, 7th and 10th days of hospitalization(C). Correlation analysis of the expression of HLA-DR and BTLA in mDCsfromseptic neonates (D) or control neonates (E).

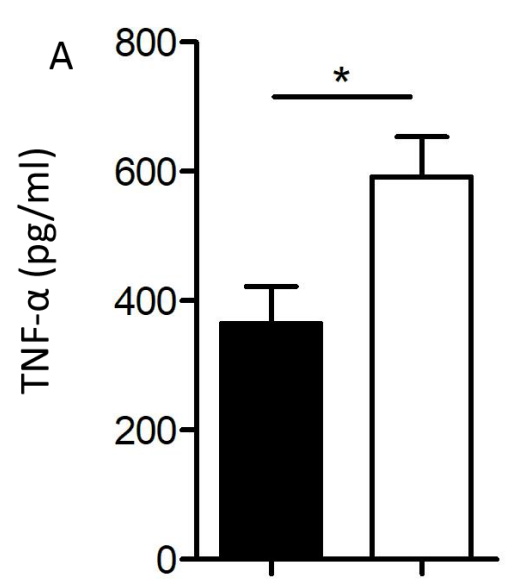

$\mathrm{BTLA}^{+} \mathrm{mDC}$ BTLA-mDC

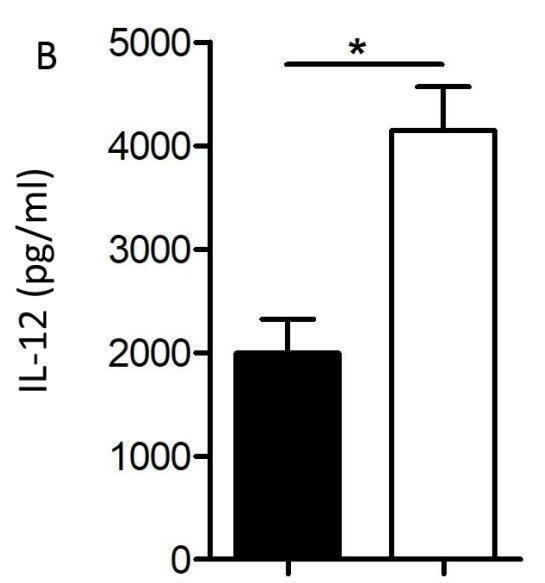

$B^{3} L^{+}{ }^{+} m D C$ BTLA-mDC

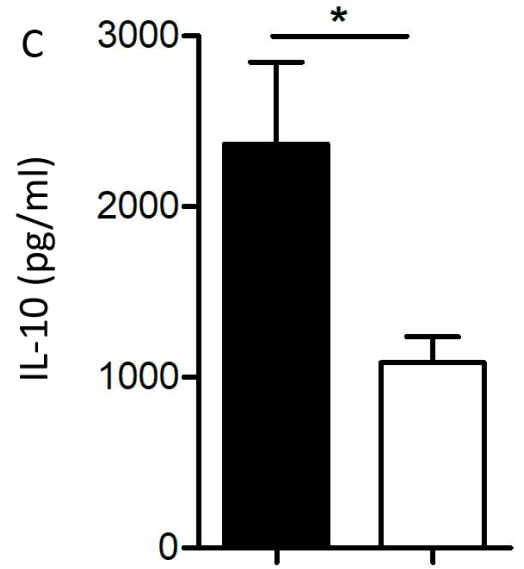

$B^{-} T^{+}{ }^{+} \mathrm{mDC}$ BTLA-mDC 
Changes of cytokine expression in BTLA-expressing mDCs. Sorted BTLA+mDCs and BTLA-mDCs from neonatal septic patients were infected with E.coli for 6h and the supernatant were collected to measure the cytokine expression with ELISA assay. Histograms showing the concentration of TNF-a (A), IL-12 (B) and IL-10 (C) between BTLA+mDCs and BTLA-mDCs. 